\title{
Alzheimer's disease qEEG \\ Spectral analysis versus coherence. Which is the best measurement?
}

\author{
Renato Anghinah', Paulo Afonso Medeiros Kanda', \\ Helder Frederico Lopes' ${ }^{1}$ Luis Fernando Hindi Basile1,3, \\ Sérgio Machado ${ }^{5}$, Pedro Ribeiro ${ }^{5}$, Bruna Velasques ${ }^{5}$, \\ Koichi Sameshima², Daniel Yasumasa Takahashi², \\ Lécio Figueira Pinto ${ }^{1}$, Paulo Caramelli ${ }^{4}$, Ricardo Nitrini'
}

\begin{abstract}
There is evidence in electroencephalography that alpha, theta and delta band oscillations reflect cognitive and memory performances and that quantitative techniques can improve the electroencephalogram (EEG) sensitivity. This paper presents the results of comparative analysis of qEEG variables as reliable markers for Alzheimer's disease (AD). We compared the sensitivity and specificity between spectral analysis (spectA) and coherence (Coh) within the same group of AD patients. SpectA and Coh were calculated from EEGs of 40 patients with mild to moderate $A D$ and 40 healthy elderly controls. The peak of spectA was smaller in the AD group than in controls. AD group showed predominance of slow spectA in theta and delta bands and a significant reduction of inter-hemispheric Coh for occipital alpha 2 and beta 1 and for frontal delta sub-band. ROC curve supported that alpha band spectA was more sensitive than coherence to differentiate controls from AD. Key words: electroencephalography, Alzheimer's disease, dementia, EEG, spectral analysis, coherence.
\end{abstract}

EEGq na doença de Alzheimer: análise espectral versus coerência. O que é melhor?

\section{RESUMO}

Há evidências de que as oscilações das bandas teta, alfa e delta no eletroencefalograma podem refletir diferenças na cognição e memória; a sensibilidade deste método diagnóstico pode ser melhorada por técnicas de quantificação. Comparamos a sensibilidade e especificidade entre a análise espectral (spectA) e coerência (Coh) dentro do mesmo grupo de pacientes com doença de Alzheimer (DA) e contra um grupo controle. SpectA e Coh foram calculadas a partir de EEGs de 40 pacientes com DA leve a moderada e 40 idosos saudáveis. O pico do espectro foi menor no grupo DA que nos controles. O grupo DA também apresentou um espectro mais lento nas bandas teta e delta e menor coerência inter-hemisférica para as sub-bandas alfa 2 e beta 1 posterior e

Correspondence

Renato Anghinah Rua Itacolomi 333 / cj 83 01239-020 São Paulo SP - Brasil

E-mail: anghinah@usp.br

\section{Support}

FAPESP, grants 03/02297-9

and 02/13633-7

Received 25 February 2011

Received in final form 4 August 2011

Accepted 11 August 2011 delta frontal. A curva ROC suporta que a análise espectral da banda alfa foi mais sensível que a coerência para diferenciar controles de DA.

Palavras-Chave: eletroencefalografia, doença de Alzheimer, demência, EEG, análise espectral, coerência.

\footnotetext{
'Departments of Neurology, School of Medicine, University of São Paulo, São Paulo SP, Brazil; ${ }^{2}$ Department of Radiology, School of Medicine, University of São Paulo, São Paulo SP, Brazil; ${ }^{3}$ Laboratory of Psychophysiology, Methodist University of São Paulo (UMESP), São Paulo SP, Brazil; ${ }^{4}$ Cognitive and Behabioral Neurology Unit, Department of Internal Medicine, Faculty of Medicine, Federal University of Minas Gerais, Belo Horizonte MG, Brazil; ${ }^{5}$ Brain Mapping and Sensory Motor Integration, Institute of Psychiatry, Federal University of Rio de Janeiro (IPUB/UFRJ), Rio de Janeiro RJ, Brazil.
} 
There is evidence in electroencephalography that alpha, theta and delta band oscillations reflect cognitive and memory performances and that quantitative techniques can improve the electroencephalogram (EEG) sensitivity ${ }^{1}$. Alzheimer's disease (AD) diagnosis should be based upon clinical history, neuropsychological and laboratory tests, neuroimaging and electroencephalography. Therefore, new approaches are necessary to enable earlier and more accurate diagnosis ${ }^{2}$ and to measure treatment results ${ }^{3}$.

EEG visual analysis is a helpful auxiliary method in $\mathrm{AD}$ diagnosis ${ }^{4}$. The most usual EEG findings are the displacement of background frequency into delta and theta ranges and the decrease or dropout of alpha central frequency ${ }^{5}$. Sandmann ${ }^{6}$ observed a direct correlation between the degree of cognitive impairment and the power of low frequency electrical activity in the EEG.

Since the first studies of quantitative EEG (qEEG) by Lehmann ${ }^{7}$ and Duffy ${ }^{8}$, spectral analysis (spectA) and other statistics have been applied to EEG. Moreover, spectA has been considered $71 \%$ to $81 \%$ sensitive to changes ${ }^{9}$ in AD EEG background. Saletu ${ }^{10}$ found a localized temporal decrease of alpha and beta activities in $\mathrm{AD}$ and a more widespread distribution of slow cerebral rhythms in vascular dementia $(\mathrm{VaD})^{11}$. Pucci ${ }^{12}$ proposed that the "alpha" rhythm could be a diagnostic AD marker, since there is a decrease in the alpha frequency to 6.0-8.0 $\mathrm{Hz}$ in mild $\mathrm{AD}$ patients.

Coherence (Coh) quantifies the covariance between pairs of signals (EEG electrodes) as a function of frequency ${ }^{13}$ and it is a well-established method to quantify connectivity through the corpus callosum ${ }^{14}$. High Coh is related to the structural and functional integrity of the intra and interhemispheric cortical connections ${ }^{15}$. Consequently, Coh is becoming widely used in $\mathrm{AD}$ studies. For example, Besthorn ${ }^{16}$ found central and frontal Coh decrease in theta, alpha and beta bands of AD patients. Studies from Locatell ${ }^{17}$ had similar results with decreased Coh in AD EEGs.

This paper compares the sensitivity and specificity of spectA versus ordinary Coh within the same group of $\mathrm{AD}$ patients and controls. The purpose is to determine the most reliable measurement in $\mathrm{AD}$ diagnosis. This approach is necessary because, most studies on AD and EEG focus on these variables without correlating them.

\section{METHOD}

We studied two groups aged from 60 to 80 years: 40 patients with mild to moderate symptoms (DSMIV-TR ${ }^{18}$ ) of probable AD (NINCDS-ADRDA criteria $\left[\right.$ McKhann $\left.{ }^{19}\right]$ ) and 40 cognitively healthy educationalmatched controls. Patients and controls were submitted to the Brazilian version of the Mini-Mental State Exam- ination (MMSE) $)^{20}$. AD patients should score below 26 points in the test.

The EEGs were recorded with the probands awake but relaxed, with closed eyes, through a twenty-electrode computer-based system (EMSA), in accordance to the 10-20 system ${ }^{21}$. Despite the fact that there are controversies regarding which reference is the best, linked ears are standard in our laboratory ${ }^{22}$. The EEG was band pass filtered for 1-30 Hz, using a sampling rate of $200 \mathrm{~Hz}$. Samples were selected by visual inspection, in order to get 20 windows of 2 seconds epochs that were free of eye blinking, drowsiness, muscle movements, or equipmentrelated artifacts. The frequency domain analysis was performed using the Fast Fourier Transform algorithm and the mean power was calculated separately within each of the sub-bands for Coh analysis (delta1: 1.0 to $<2.0$ Hz; delta2: 2.0 to $<4.0 \mathrm{~Hz}$; theta1: 4.0 to $<6.0 \mathrm{~Hz}$; theta2: 6.0 to $<8.0 \mathrm{~Hz}$; alpha1: 8.0 to $<10.0 \mathrm{~Hz}$; alpha2: 10.0 to $<12.5 \mathrm{~Hz}$; beta1: 12.5 to $<15.0 \mathrm{~Hz}$; beta2: 15.0 to $<20$ $\mathrm{Hz}$ and beta3: 20.0 to $\leq 30$ ). Interhemispheric Coherence (IHCoh) from EEG pairs of electrodes were performed in the original referential montage (Fp1-Fp2; F7-F8; F3F4; C3-C4; P3-P4; T5-T6 and O1-O2) for EEG bands and sub-bands. We did not "window" the FFT in any additional way. IHCoh was calculated following the formula: $\operatorname{Coh}(f) 2=[\operatorname{Gxy}(f)] 2 / \operatorname{Gxx}(f) \operatorname{Gyy}(f)$.

The spectA peak was calculated from the maximum spectral power (not the mean frequency) found in each frequency band. The average Coh of controls and $\mathrm{AD}$ was statistically compared (Student t-test) and Pearson correlation was also calculated for the inter-hemispheric Coh among all electrodes to each other, in all bands and in both groups. Furthermore, Receiver Operating Characteristic Curve (ROC) analysis was used to compare the accuracy of the EEG variables to discriminate AD patients from controls.

\section{RESULTS}

The AD group spectA peak showed a slow bands increase (delta and theta) and an alpha band decrease mainly in posterior areas (T5-T6; O1-O2). The AD group spect A frequency peak was significantly reduced to the range from 6.0 to $10.2 \mathrm{~Hz}$. Controls had values from 8.1 to $10.9 \mathrm{~Hz}$ ( $\mathrm{t}$ test for independent samples; $\mathrm{p}<0.001$ ). Coh analysis also revealed significant differences between the two groups (t-test) for the following electrode pairs and frequencies: Fp1-Fp2: delta1 ( $\mathrm{p}=0.022)$; F3-F4: delta2 $(\mathrm{p}=0.033)$; alpha1 $(\mathrm{p}=0.004)$; alpha2 $(\mathrm{p}=0.002)$; beta 1 $(\mathrm{p}=0.001)$; beta2 $(\mathrm{p}=0.008) ; \mathrm{C} 3-\mathrm{C} 4$ : beta1 $(\mathrm{p}=0.046)$; P3-P4 alpha1 ( $\mathrm{p}=0.030)$; O1-O2: alpha1 ( $\mathrm{p}=0.026)$.

Roc analysis was calculated for all band in all groups of electrodes. Alpha1 spectA peak and alpha1 inter-hemispheric Coh showed the two biggest areas under the Roc 


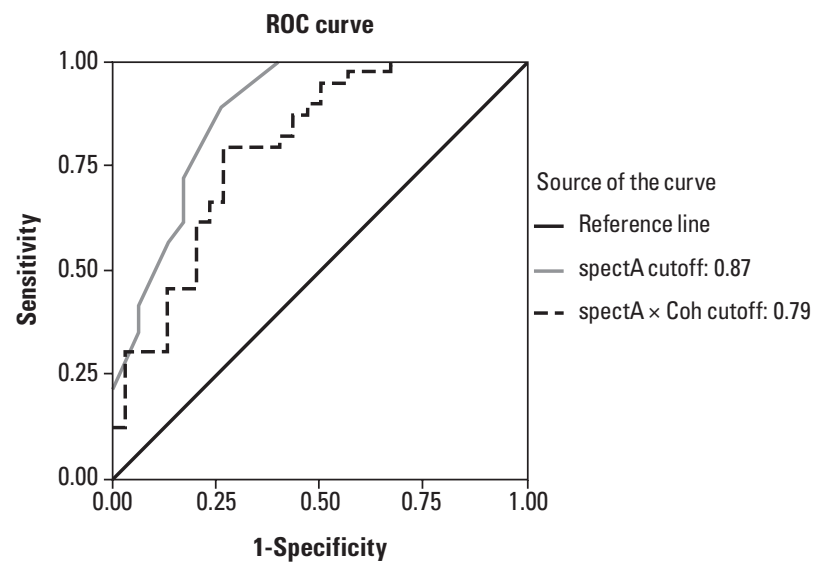

Fig 1. ROC (Receiver Operating Characteristic Curve). The performance of a diagnostic variable can be quantified by calculating the area under the ROC curve (AUROC). The ideal test would have an $A U R O C$ of 1 , whereas a random guess would have an AUROC of $0.5^{28}$

curve (related to posterior electrodes: O1, O2). Consequently, we discarded the additional information and we only compared alpha1 spectA peak to alpha1 inter-hemispheric Coh (Fig 1). When both were considered, the area under the curve was 0.79 . Considered alone spectA peak area under its ROC curve was 0.87 . Therefore, in this specific sample of EEGs, spectA was more sensitive and specific than Coh to discriminate $\mathrm{AD}$ patients.

The Pearson correlation analysis showed lower correlations between fast frequencies in controls compared with AD patients (Fig 2A and 2B). This comparison showed that the significant correlation in the $\mathrm{AD}$ group occurred mostly in the anterior and medium scalp areas to delta and theta frequency bands. In the control group, the significant correlation was more diffusely distributed through all areas and frequency bands, showing correlation between alpha and beta bands in the posterior and medium scalp areas and between delta and theta bands in the more anterior part of the scalp.

\section{DISCUSSION}

Possibly our findings are related to a broader dementia spectrum than to AD. However, subjects were recruited for inclusion in this study from University of São Paulo's Alzheimer's ambulatory (CEREDIC). Consequently, our findings reflect only our sample behaviour. We probably would find similar results comparing normals with other dementia subgroups and the specificity would be much lower comparing AD patients with other neurodegenerative or psychiatric diseases. These are questions to be answered in the future.

Eye movements and blink contamination are pervasive problems in EEG research. Their electric potentials can propagate across much of the scalp distorting brain
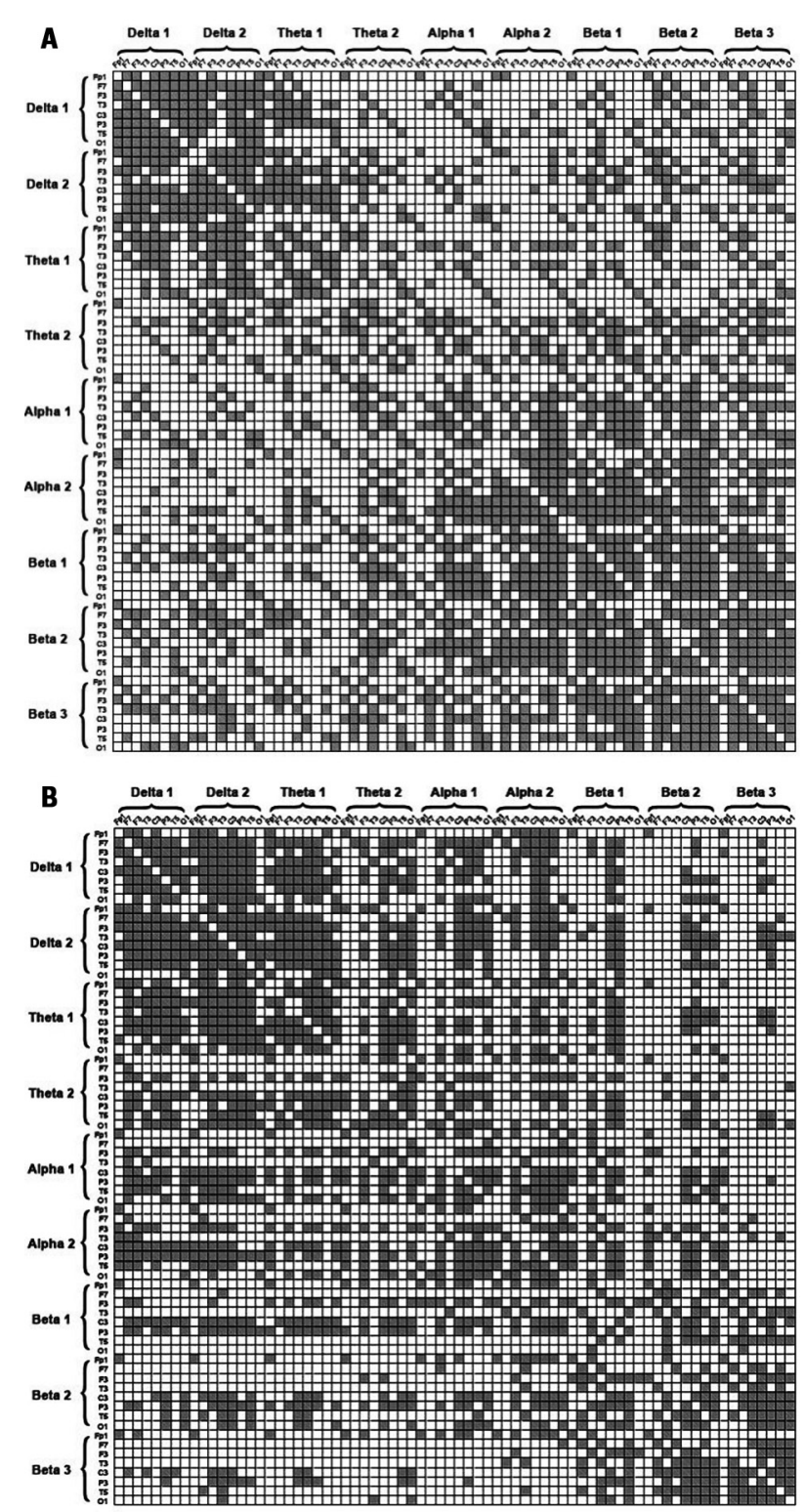

Fig 2. Coh values (Pearson correlation) between homologous electrode pairs (left-right sides)for all spectral bands and areas. Electrode pairs are labeled only by their left side designation (ex: $\mathrm{Fp} 1=\mathrm{Fp} 1+\mathrm{Fp} 2 ; \mathrm{F} 7=\mathrm{F} 7+\mathrm{F} 8 ; \mathrm{F} 3=\mathrm{F} 3+\mathrm{F} 4$; etc). The entries colored in grey correspond to statistically significant correlation values (ssv) $(p<0.05)$. [A] Control group: ssv was more diffusely distributed showing correlation between alpha and beta bands in the posterior and medium scalp areas and between delta and theta bands in the more anterior part of the scalp; [B] Alzheimer's disease: ssv occurred mostly in the anterior and medium scalp areas to delta and theta frequency bands.

signals. To cope with these artifacts we used short EEG FFT epochs to increase the likelihood that there was artifact-free data available for analysis in $\mathrm{AD}$ patients (some of them poorly cooperative).

The EEG alpha1 spectA peak differed clearly between $\mathrm{AD}$ group and controls, with a control group cut-off at $8.0 \mathrm{~Hz}$. This result is in agreement with previous studies 
which concluded that values of alpha peak below $8.0 \mathrm{~Hz}$ in EEG background may be considered pathological ${ }^{23}$.

The importance of alpha spectA analysis is here emphasized because alpha rhythm constitutes the background of the normal EEG in awake individuals and its slowing is associated with brain dysfunction ${ }^{24}$. For example, two parameters related to central areas have been recently considered as markers of the progression of $\mathrm{AD}$ : alpha rhythm frequency decrease and theta power increase $^{24}$. Our results are consistent with Dringenberg findings that cholinergic and monoaminergic decrease occurs in $\mathrm{AD}$ resulting in a EEG background activity displacement to lower frequencies ${ }^{25}$.

The inter-hemispheric Coh was statistically reduced for $\mathrm{AD}$ group in two areas of the scalp: alpha 2 and betha1 sub-bands in posterior areas and delta and theta in anterior areas. These results can be explained by a decrease in overall cortical connections as a consequence of neuronal loss in $\mathrm{AD}^{26}$. This theory is supported by Holschneider's ${ }^{27}$ studies in rats, which demonstrated that Coh is sensitive to cholinergic deafferentation, particularly to the loss of long cortico-cortical connections. This latter study is in accordance with our findings of significantly reduced Pearson correlation coefficients in AD versus the control group.

The novelty of the present work lies in the comparison between the sensitivity and specificity between Coh and spectA within the same group of $\mathrm{AD}$ patients. In this cohort of patients we found that spectA was more sensitive and specific than Coh to discriminate $\mathrm{AD}$, though both methods can be helpful when added to AD diagnostic protocols. Nevertheless, the definite inclusion of qEEG in clinical practice awaits a systematic replication and validation of our results.

\section{REFERENCES}

1. Fonseca L, Tedrus G, Letro G, Bossoni A. Dementia, mild cognitive impairment and quantitative EEG in patients with Parkinson's disease. Clin EEG Neurosci 2009:40:168-172.

2. Martinez ALD, Giacobini E, Greig NH. Advances in Alzheimer therapy: understanding pharmacological approaches to the disease. Curr Alzheimer Res 2009;6:83-85.

3. Sneddon R, Shankle WR, Hara J, Rodriquez A, Hoffman D, Saha U. qEEG monitoring of Alzheimer's disease treatment: a preliminary report of three case studies. Clin EEG Neurosci 2006;37:54-59.

4. Luccas FJ, Anghinah R, Braga Nl, et al. Guidelines for recording/analyzing quantitative EEG and evoked potentials. Part II: Clinical aspects. Arq Neuropsiquiatr 1999;57:132-146.

5. Klass D, Brenner R. Electroncephalography of the elderly. J Clin Neurophysiol 1995;12:116-131.
6. Sandmann M, Piana E, Sousa D, Bittencourt P. Digital EEG with brain mapping in Alzheimer's dementia and Parkinson's disease. A prospective controlled study. Arq Neuropsiquiatr 1996;54:50-56.

7. Lehmann D. Multichannel topography of human alpha EEG fields. Electroenceph Clin Neurophysiol 1971;31:439-449.

8. Duffy F, Burchfiel J, Lombroso C. Brain electrical activity mapping (BEAM): a method for extending the clinical utility of EEG and evoked potential data. Ann Neurol 1979;5:309-321.

9. Martin-Loeches M, Gil P, Jimenez F, et al. Topographic maps of brain electrical activity in primary degenerative dementia of Alzheimer type and multiinfarct dementia. Biol Psych 1991;29:211-223.

10. Saletu B, Paulus E, Grunbergerer J. Correlation maps: on the relation of electroencephalographic slow wave activity to computerized tomography and psycopathometric measurements in dementia. Imaging of Brain in Psychiatry and Related Fieldsed. Berlin;Springer-Verlag 1993:263-265.

11. Martin-Loeches M, Garcia-Trapero J, Gil P, Rubia FJ. Topography of mobility and complexity parameters of the EEG in Alzheimer's disease. Biol Psychiatry 1991;30:1111-1121.

12. Pucci E, Belardinelli N, Cacchiò G, Signorino M, Angeleri F. EEG power spectrum differences in early and late onset forms of Alzheimer's disease. Clin Neurophysiol 1999;110:621-631.

13. Shaw JC. An introduction to the coherence function and its use in EEG signal analysis. J Med Eng and Tech 1981;5:279-288.

14. Nielsen TMJ, Lassonde M. Decreased Interhemisferic EEG Coherene during sleep in agenesis of the corpus calosum. Eur Neurol 1993;33:173-176.

15. Claus JJ, Strijers RL, Jonkman EJ, et al. The diagnostic value of electroencephalography in mild senile Alzheimer's disease. Clin Neurophysiol 1999;110:825-832.

16. Besthorn C, Zerfass R, Geiger-Kabisch C, et al. Discrimination of Alzheimer's disease and normal aging by EEG data. Electroencephalogr Clin Neurophysiol 1997; 103:241-248.

17. Locatelli T, Cursi M, Liberati D, Franceschi M, Comi G. EEG coherence in Alzheimer's disease. Electroencephalogr Clin Neurophysiol 1998;106: 229-237.

18. Diagnostic and Statistical Manual (DSM-IV-TR). Washington DC: American Psychiatric Association; 2000.

19. McKhann G, Drachman D, Folstein M, Katzman R, Price D, Stadlan EM. Clinical diagnosis of AD: report of the NINCDS-ADRDA work group under the auspices of Department oh health and human services task force on AD. Neurology 1984;34:939-944.

20. Brucki S, Nitrini R, Caramelli P, Bertolucci P, Okamoto I. Suggestions for utilization of the mini-mental state examination in Brazil. Arq Neuropsiquiatr 2003;61:777-781.

21. Jasper $\mathrm{H}$. The ten-twenty electrode system of the International Federation. Electroencephalogr Clin Neurophysiol 1958;10:371-373.

22. Rappelsberger P. EEG coherence and reference signals: experimental results and mathematical explanations. Med BiolEng Comput 1998;38: 399-406.

23. Niedermeyer E, Lopes da Silva F. The normal EEG of the waking adult. In: Electroencephalography: Basic Principles, Clinical Applications and Related Fields. Lippincott Williams \& Wilkins;2005.

24. Jelic V. Early diagnosis of Alzheimer Disease. Phd Thesis. Karolinska Institutet, Stockholm; 1999:1-14

25. Dringenberg H. Alzheimer disease: more than a cholinergic diosorder evidence that cholinergicmonoaminergic interactions contribute to EEG slowing and dementia. Behav Brain Research 2000;115:235-249.

26. Kuks JB, Vos JE, O'Brien MJ. Coherence patterns of the infant sleep EEG in absence of the corpus callosum. Electroencephalogr Clin Neurophysiol 1987;66:8-14.

27. Holschneider DP, Leuchter AF, Scremin OU, Treiman DM, Walton NY. Effects of cholinergic deafferentation and NGF on brain electrical coherence. Brain Res Bull 1998;45:531-541.

28. Bewick V, Cheek L, Ball J. Statistics review 13: receiver operating characteristic curves. Crit Care 2004:8:508-512. 